\title{
Nonsense-mediated mRNA decay affects nonsense transcript levels and governs response of cystic fibrosis patients to gentamicin
}

\author{
Liat Linde, ${ }^{1}$ Stephanie Boelz, ${ }^{2,3}$ Malka Nissim-Rafinia, ${ }^{1}$ Yifat S. Oren, ${ }^{1}$ Michael Wilschanski, 4
} Yasmin Yaacov, ${ }^{4}$ Dov Virgilis, ${ }^{5}$ Gabriele Neu-Yilik, ${ }^{2,3}$ Andreas E. Kulozik, ${ }^{2,3}$ Eitan Kerem, ${ }^{4}$ and Batsheva Kerem ${ }^{1}$

\begin{abstract}
1Department of Genetics, Life Sciences Institute, The Hebrew University of Jerusalem, Jerusalem, Israel. ${ }^{2}$ Molecular Medicine Partnership Unit, University of Heidelberg and European Molecular Biology Laboratory, Heidelberg, Germany. ${ }^{3}$ Department for Pediatric Oncology, Hematology, and Immunology, University Hospital Heidelberg, Heidelberg, Germany. ${ }^{4} \mathrm{CF}$ Center, Hadassah University Hospital, Mount Scopus, Jerusalem, Israel. ${ }^{5}$ Department of Pediatrics,
\end{abstract} Shaare Zedek Medical Center, Jerusalem, Israel.

\begin{abstract}
Aminoglycosides can readthrough premature termination codons (PTCs), permitting translation of fulllength proteins. Previously we have found variable efficiency of readthrough in response to the aminoglycoside gentamicin among cystic fibrosis (CF) patients, all carrying the W1282X nonsense mutation. Here we demonstrate that there are patients in whom the level of CF transmembrane conductance regulator (CFTR) nonsense transcripts is markedly reduced, while in others it is significantly higher. Response to gentamicin was found only in patients with the higher level. We further investigated the possibility that the nonsense-mediated mRNA decay (NMD) might vary among cells and hence governs the level of nonsense transcripts available for readthrough. Our results demonstrate differences in NMD efficiency of CFTR transcripts carrying the W1282X mutation among different epithelial cell lines derived from the same tissue. Variability was also found for 5 physiologic NMD substrates, RPL3, SC35 $1.6 \mathrm{~kb}$, SC35 $1.7 \mathrm{~kb}, A S N S$, and CARS. Importantly, our results demonstrate the existence of cells in which NMD of all transcripts was efficient and others in which the NMD was less efficient. Downregulation of NMD in cells carrying the W1282X mutation increased the level of CFTR nonsense transcripts and enhanced the CFTR chloride channel activity in response to gentamicin. Together our results suggest that the efficiency of NMD might vary and hence have an important role in governing the response to treatments aiming to promote readthrough of PTCs in many genetic diseases.
\end{abstract}

\section{Introduction}

Many inherited diseases result from nonsense mutations leading to premature termination codons (PTCs) (1). One therapeutic approach for patients carrying in-frame nonsense mutations is aimed at promoting readthrough of the PTCs to enable the expression of full-length, functional proteins $(2,3)$. Studies in tissue culture cells as well as in mouse models have shown that aminoglycosides can readthrough disease-causing PTCs and partially restore the expression and/or the function of these proteins (4-19). Further clinical trials in Duchenne muscular dystrophy (DMD) $(20,21)$, Becker muscular dystrophy (20), and cystic fibrosis $(\mathrm{CF})(22,23)$ patients have shown that aminoglycosides can promote in vivo readthrough of nonsense mutations and lead to expression of full-length proteins and/or correction of the protein function. However, in several studies, no expression of full-length functional proteins was actually observed, suggesting no response to the aminoglycoside treatment $(15,16,21-25)$. This variability

Nonstandard abbreviations used: ASNS, asparagine synthetase; CARS, cysteinyl-tRNA synthetase; $\mathrm{CF}$, cystic fibrosis; $\mathrm{CFTR}, \mathrm{CF}$ transmembrane conductance regulator; $\mathrm{CHX}$, cycloheximide; KRT18, keratin 18; NMD, nonsense-mediated mRNA decay; NPD, nasal potential difference; PTC, premature termination codon; RPL3, ribosomal protein L3; RPS9, ribosomal protein S9; SC35, splicing component 35 kDa; UPF1, UPF1 regulator of nonsense transcripts homolog (yeast).

Conflict of interest: The authors have declared that no conflict of interest exists. Citation for this article: J. Clin. Invest. 117:683-692 (2007). doi:10.1172/JCI28523. in response was proposed to be associated with the identity of the PTC and its sequence context (26-28), as well as with the chemical composition of the aminoglycoside, the duration of treatment, and the method of application.

We have recently administered gentamicin to the nasal epithelium of CF patients, all of whom carried the same W1282X nonsense mutation (29). This mutation is associated with a severe form of the CF disease (30). In many of the patients, improvement of the typical CF electrophysiological abnormalities was found and expression of full-length $C F$ transmembrane conductance regulator (CFTR) proteins was demonstrated following the treatment. In other patients, however, no correction of the electrophysiological abnormalities was found, indicating no response to gentamicin. The reasons for this variable response remained unknown. Since the templates for readthrough treatment are the transcripts carrying a PTC, we hypothesized that the level of these transcripts is a limiting factor in the response.

PTC-bearing transcripts are detected and degraded by the nonsense-mediated mRNA decay (NMD) pathway, a posttranscriptional translation-dependent surveillance mechanism that prevents the synthesis of proteins carrying PTCs $(31,32)$. NMD has been shown to degrade transcripts carrying disease-causing nonsense or frameshift mutations, as well as a variety of physiologic transcripts. Among these are transcripts with upstream open reading frame (uORF), transcripts containing introns in the 
A

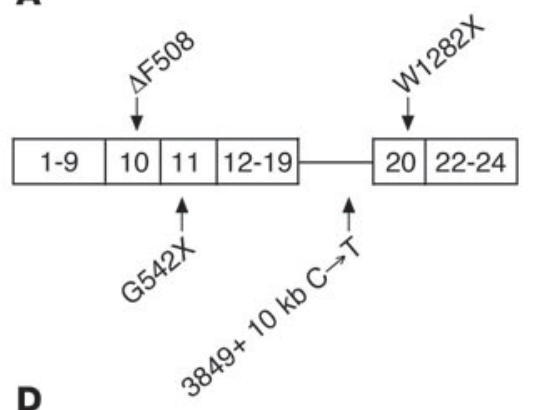

B

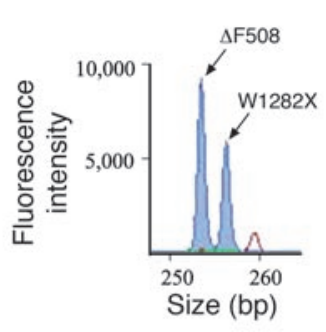

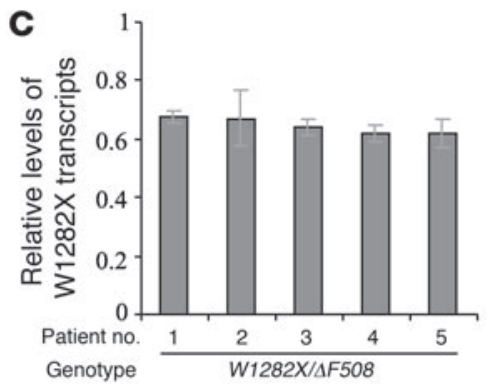

$\mathbf{E}$

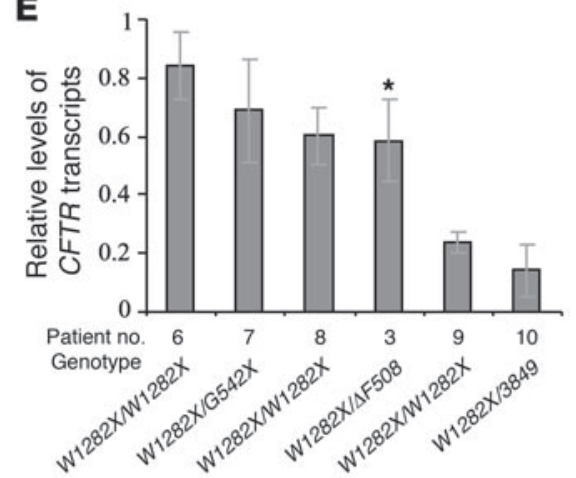

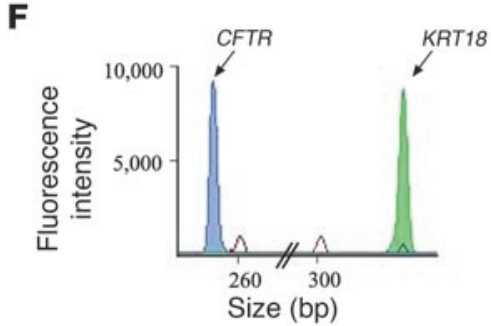
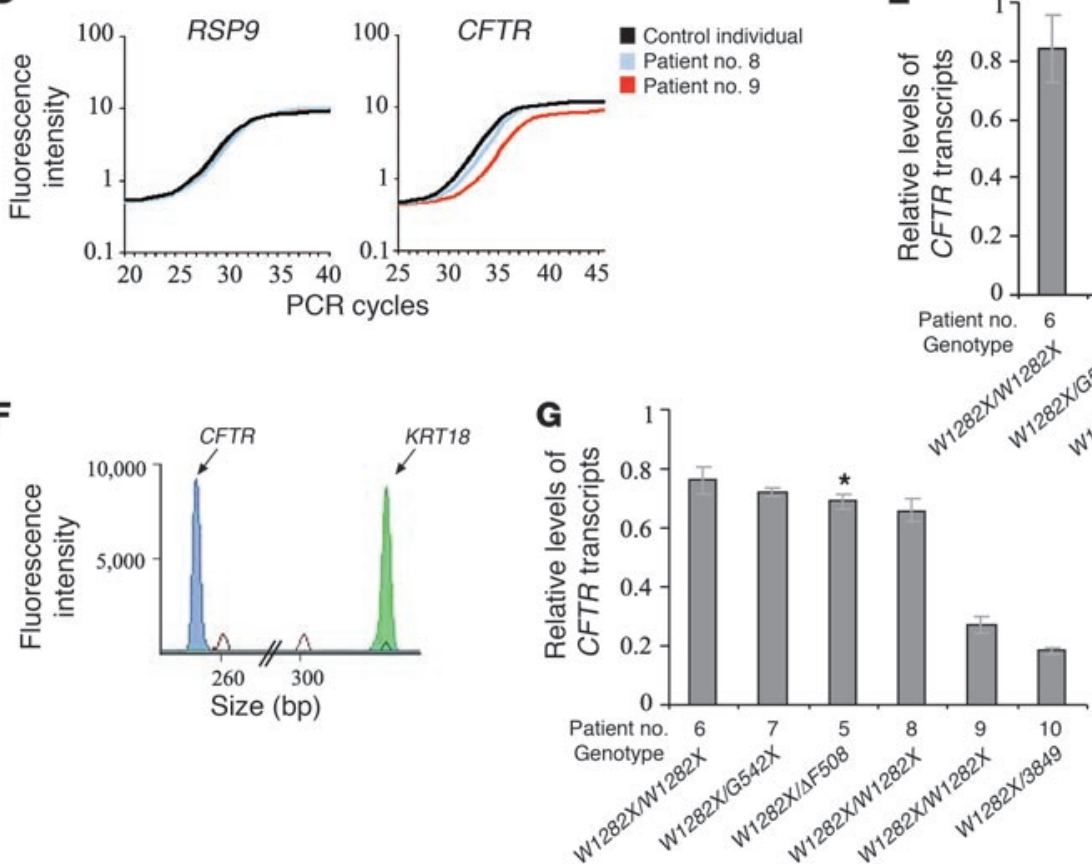

\section{Figure 1}

Levels of CFTR nonsense transcripts in nasal epithelium of CF patients. (A) A scheme of localization of the CFTR mutations carried by the studied patients. The numbers within the boxes mark the CFTR exons. The connecting horizontal line marks intron 19. (B) An example of GeneScan analysis of RT-PCR products from the $\Delta$ F508 region (patient 3). The size marker appears as a red peak (260 bp). (C) A summary of the W1282X transcript levels in the $W 1282 X / \Delta F 508$ patients. These levels were compared to the level of $\Delta F 508$ transcripts. (D) An example of real-time PCR of the CFTR and the RPS9 genes, of RNA from 2 patients (nos. 8 and 9) homozygous for W1282X and a control individual with normal CFTR alleles. (E) A summary of the CFTR transcript levels, in the patients carrying 2 nonsense mutations. The levels were normalized to the level of RPS9 and compared to the normalized level obtained from a control individual with normal CFTR alleles. The asterisk marks the CFTR transcript level in patient 3, who was also analyzed as described in C, for comparison of the results of the GeneScan and real-time PCR analyses. (F) An example of GeneScan analysis of RT-PCR products of the CFTR and the KRT18, in a patient homozygous for the W1282X mutation (patient 6). The size marker appears as red peaks (260 bp and $300 \mathrm{bp}$ ). (G) A summary of the CFTR transcript levels in the patients carrying 2 nonsense mutations. These levels were normalized to the level of KRT18 and compared to the normalized level obtained from a control individual with normal CFTR alleles. The asterisk marks the CFTR transcript level in patient 5, who was also analyzed as described in C, for comparison of the GeneScan results. The levels in $\mathbf{C}, \mathbf{E}$, and $\mathbf{G}$, are shown as mean \pm SEM.

$3^{\prime}$ untranslated region (UTR), and transcripts derived from alternative splicing (33-37).

Here we have investigated the possibility that NMD efficiency might vary among different cells and hence affects the level of PTC-bearing transcripts available for readthrough treatment. We demonstrate that the same PTC can elicit NMD with variable efficiency among cells derived from nasal epithelium. Variability in the NMD efficiency was found for CFTR transcripts carrying the W1282X mutation as well as for several physiologic NMD substrates, ribosomal protein L3 (RPL3), splicing component $35 \mathrm{kDa}$ (SC35) $1.6 k b, S C 351.7 \mathrm{~kb}$, asparagine synthetase (ASNS), and cysteinyl-tRNA synthetase (CARS). We further showed that downregulation of NMD increased the level of CFTR nonsense transcripts and led to enhanced CFTR chloride channel activity in response to gentamicin. Together, our results suggest that the efficiency of NMD may vary among cell lines and hence affect the level of transcripts carrying PTCs, and govern the response to readthrough treatment.

\section{Results}

Different levels of nonsense CFTR $m R N A$ in samples from patients. In order to investigate the possibility that the response of patients to readthrough treatment is associated with the level of mRNA tran- 


\section{Table 1}

Study patients and response to gentamicin treatment

\begin{tabular}{|c|c|c|c|c|c|c|}
\hline \multirow{2}{*}{$\begin{array}{l}\text { Patient } \\
\text { number }\end{array}$} & \multirow[t]{2}{*}{ Genotype } & \multicolumn{2}{|c|}{ Basal NPD } & \multicolumn{2}{|c|}{ Chloride transport } & \multirow[t]{2}{*}{ Response } \\
\hline & & Before treatment & After gentamicin & Before treatment & After gentamicin & \\
\hline 1 & $W 1282 X / \Delta F 508$ & -38 & -18 & -1 & -6 & + \\
\hline 2 & $W 1282 X / \Delta F 508$ & -41 & -41 & -3 & -5 & + \\
\hline 3 & $W 1282 X / \Delta F 508$ & -43 & -30 & -1 & -4 & + \\
\hline 4 & $W 1282 X / \Delta F 508$ & -40 & -30 & 4 & -4 & + \\
\hline 5 & $W 1282 X / \Delta F 508$ & -40 & -30 & -2 & -2 & $+/-A$ \\
\hline 6 & $W 1282 X / W 1282 X$ & -47 & -32 & 0 & -9 & + \\
\hline 7 & W1282X/G542X & -43 & -45 & -2 & -11 & + \\
\hline 8 & W1282X/W1282X & -40 & -35 & 11 & -9 & + \\
\hline 9 & W1282X/W1282X & -32 & -33 & 2 & 4 & - \\
\hline 10 & $W 1282 X / 3849+10 \mathrm{~kb} C \rightarrow T$ & -65 & -56 & 3 & -3 & - \\
\hline
\end{tabular}

All values are expressed as $\mathrm{mV}$. The normal range for basal potential difference in our laboratory is $\geq-30 \mathrm{mV}$, and the normal range of chloride transport is $\leq-4 \mathrm{mV}(40)$. Aln all previous clinical trials in CF patients, aminoglycosides $(22,23,29)$ or other molecules $(41-44)$ influenced only the chloride transport abnormality. In this patient, there was no change in chloride transport, while normalization of basal potential difference was observed. This is unusual, and hence the response of this patient remained inconclusive (+/-).

scripts carrying the W1282X mutation, we obtained RNA samples from nasal epithelial cells from patients who had participated in our double-blind, placebo-controlled, crossover trial (29). The level of nonsense CFTR mRNA was analyzed in pretreatment samples from these patients. Five of the patients were heterozygous for the W1282X and the $\Delta \mathrm{F} 508$ mutations (Figure $1 \mathrm{~A}$ ). The $\Delta \mathrm{F} 508$ mutation is a 3-bp deletion, which is not expected to be affected by NMD, hence the level of $\Delta \mathrm{F} 508$ transcripts is similar to that of wild-type (38). Thus, the level of mRNA transcribed from W1282X alleles could be distinguished and compared to those transcribed from the $\Delta F 508$ allele. The results showed that the level of the W1282X mRNA among these patients was reduced to between $62 \% \pm 0.05 \%$ and $68 \% \pm 0.02 \%$ of that transcribed from the $\Delta \mathrm{F} 508$ allele (Figure 1, B and C). The other 5 patients carried 2 alleles with CFTR nonsense mutations, of which at least 1 was the W1282X (Figure 1A). The CFTR mRNA levels in these patients were normalized to those transcribed from a control gene, the ribosomal protein S9 (RPS9), which we found to be expressed at the same level in all tested individuals and whose expression was not affected by the gentamicin treatment. The level of the normalized CFTR nonsense mRNA in each patient was compared to the normalized level obtained from a control individual with normal CFTR alleles. The results revealed that in 3 patients (nos. 6, 7, and 8), the level of CFTR nonsense mRNA was between $60 \% \pm 0.10 \%$ and $84 \% \pm 0.12 \%$ of that found in a control individual with normal CFTR alleles, similar to the level found in the $W 1282 X / \Delta F 508$ patients (Figure $1, \mathrm{D}$ and $\mathrm{E}$ ). In contrast, in 2 patients (nos. 9 and 10), the level was considerably lower, $24 \% \pm 0.04 \%$ and $14 \% \pm 0.09 \%$, respectively. We further analyzed 1 patient heterozygous for the W1282X and the $\Delta$ F508 mutations (patient 3) by both GeneScan and real-time PCR analyses for comparisons of the results obtained by the different mRNA quantification methods. As shown in Figure 1, C and E, similar levels were observed. Furthermore, 2 of the patients homozygous for W1282X (nos. 8 and 9) are sisters whose parents are first-degree cousins. Although they carry identical CFTR alleles, there was a large difference in their levels of W1282X transcripts. Patient 8 had $60 \% \pm 0.10 \%$ of the level found in a control individual, while patient 9 had $24 \% \pm 0.04 \%$. Consistent results were obtained in RNA samples derived from these patients at various time points (Supplemental Figure 1; supplemental material avail- able online with this article; doi:10.1172/JCI28523DS1). In order to exclude the possibility that the observed variability resulted from variation in the representation of epithelial cells in the samples, we further normalized the level of CFTR transcripts in these patients to that of keratin 18 (KRT18), a marker of ciliated and secretory epithelial cells (39), which we found not affected by the gentamicin treatment. The level of the normalized CFTR nonsense transcripts in each patient was compared to the normalized level obtained for a control individual with normal CFTR alleles. The results revealed normalized levels similar to those obtained with RPS9 (Figure 1, F and G). It is important to note that no change in the level of nonsense transcripts was found in any of the patients following the placebo or the gentamicin treatment (Supplemental Figure $2 \mathrm{~A}$ ). Together, the results indicate that our patients can be categorized into 2 groups, one in which the level of CFTR nonsense transcripts is markedly reduced and the other in which the level is significantly higher.

In order to investigate the role of transcription efficiency in regulating the level of CFTR mRNA, we analyzed the level of CFTR pre-mRNA in 4 patients (nos. 2, 5, 8, and 10). The results showed that the relative CFTR pre-mRNA levels were not significantly different $(P=0.189)$ among the patients (Supplemental Figure 3). Hence, the markedly reduced level of nonsense CFTR mRNA in patient 10 (shown in Figure 1) was not the result of lower CFTR transcription efficiency.

The level of CFTR nonsense transcripts is associated with the response of patients to gentamicin treatment. We further analyzed whether the relative level of CFTR nonsense transcripts is associated with the response of the patients to gentamicin treatment. As shown in Table 1, normalization of the abnormal chloride transport $(\leq-4$ $\mathrm{mV}$ ) was found in 7 of the 8 patients with the higher level of CFTR nonsense transcripts. In some of these patients (nos. 1, 3, and 4), the normalization of chloride transport was associated with improvement toward normalization of the basal nasal potential difference (NPD) ( $\geq-30 \mathrm{mV}$ ) (40). The reason for this improvement in a subset of the patients is unclear. In one patient, patient 5 , there was no change in chloride transport, while normalization of basal NPD was observed. It is important to note that in all previous clinical trials in CF patients, aminoglycosides $(22,23,29)$ or other molecules (41-44) affected only the chloride transport 

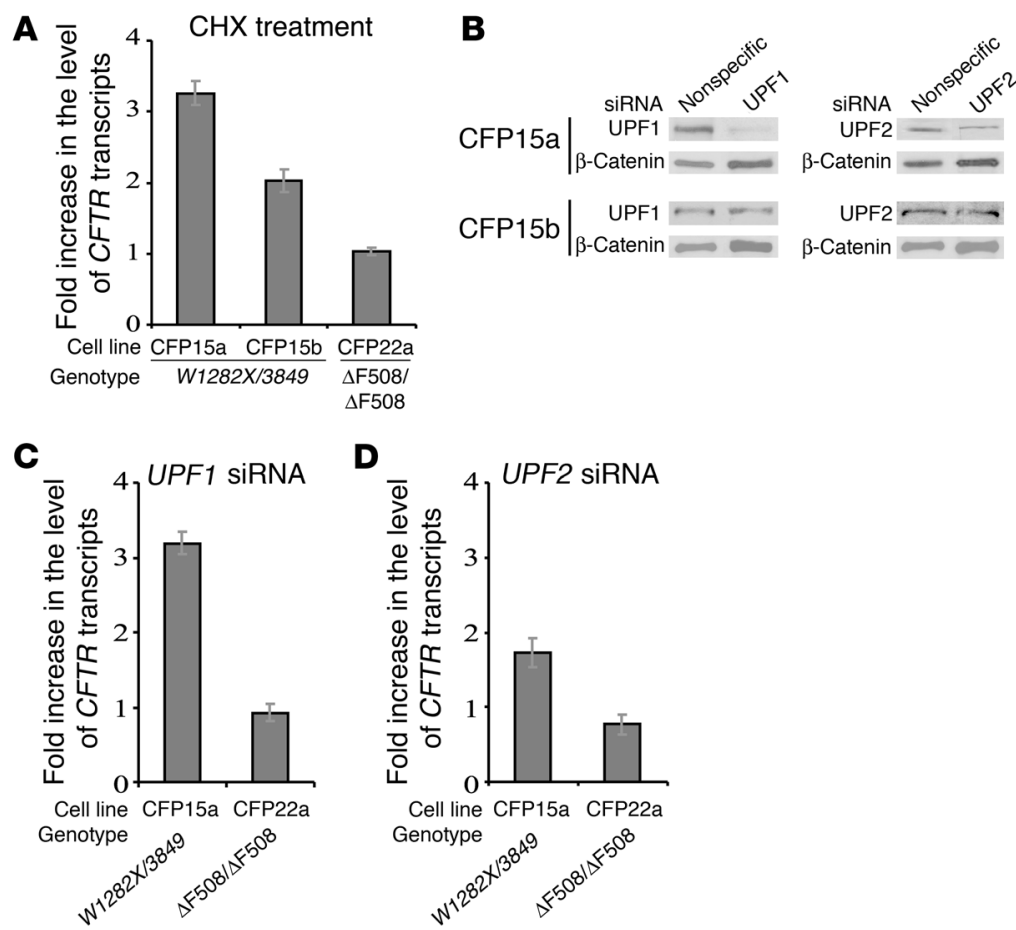

abnormality; hence, the response of patient 5 remained inconclusive. No response was found in the patients with markedly reduced transcript levels (patients 9 and 10). Together, the results suggest that the level of nonsense transcripts might be a limiting factor in readthrough of PTCs in response to gentamicin treatment, although the variability in CFTR transcript level cannot entirely explain the variability in the response.

Variable NMD efficiency of endogenous CFTR transcripts carrying the W1282X mutation. Previous studies showed that the level of W1282X transcripts is markedly reduced in epithelial samples derived from CF patients and in a bronchial epithelial cell line, IB3-1, heterozygous for the W1282X and the $\Delta$ F508 mutations (5, 45). These results raised the possibility that transcripts carrying the W1282X mutation are subject to NMD. PTCs that are located more than 50-55 nucleotides upstream of the final exon-exon junction can induce NMD (46). Since the last CFTR exon-exon junction is located more than 55 nucleotides downstream to the W1282X mutation, transcripts carrying the W1282X mutation have the potential to be subject to NMD. In order to investigate this possibility, we analyzed nasal epithelial cell lines (CFP15a and CFP15b) derived from polyps of 2 unrelated CF patients, both heterozygous for the W1282X and the $3849+10 \mathrm{~kb} \mathrm{C} \rightarrow \mathrm{T}$ mutations. The latter is a change from $\mathrm{C}$ to $\mathrm{T}$ that generates a splicing donor site that can lead to inclusion of an 84-bp cryptic exon harboring a PTC (47). We examined the level of CFTR nonsense transcripts following treatment with cycloheximide (CHX), a known indirect inhibitor of NMD (48). The CFTR transcript level was normalized to that of RPS9, and the ratio between these normalized levels following CHX treatment was calculated and compared to the ratio in untreated cells. Following CHX treatment in CFP15a cells, a $3.3 \pm 0.16$-fold increase in the CFTR transcript level relative to untreated cells was found, while in CFP15b the increase was only $2.0 \pm 0.16$-fold (Figure 2A). These results suggest that endogenous CFTR W1282X transcripts might be subject to NMD with variable

\section{Figure 2}

Effect of NMD inhibition on the level of endogenous CFTR transcripts. (A) Effect of indirect NMD inhibition by $\mathrm{CHX}$ on the level of CFTR transcripts. The level was measured by real-time PCR and normalized to that of RPS9 and the ratio between these normalized levels following treatment was calculated and compared to the ratio in untreated cells. (B) Western blot analysis in CFP15a (upper panels) and CFP15b (lower panel) cells following sequence-specific downregulation of UPF1 and UPF2. (C and D) Effect of direct NMD inhibition by siRNA directed against UPF1 (C) and UPF2 (D) on the level of CFTR transcript. The level was measured and normalized as described in $\mathbf{A}$. The increase in the level of CFTR transcripts is shown as mean \pm SEM.

efficiencies. As a control, we administered CHX to another nasal epithelial cell line (CFP22a), which is homozygous for the $\Delta \mathrm{F} 508$ mutation. No change in the CFTR transcript level was found in these cells (Figure $2 \mathrm{~A}$ ). This indicated that the inhibition of NMD by $\mathrm{CHX}$ is specific to transcripts carrying PTCs.

To further study the mechanism of this variability, we tested the effect of siRNA duplexes on UPF1 and UPF2 (UPF1 and -2 regulator of nonsesnse transcripts homo$\log$ (yeast); also known as RENT1 and RENT2, respectively). These factors are essential for the NMD pathway (49). As a control, we analyzed the effect of nonspecific siRNA duplexes. Western blot analysis in CFP15a cells showed a sequence-specific downregulation of both UPF1 and UPF2. However, the downregulation of UPF1 was more efficient (Figure 2B, upper panels). Western blot analysis in CFP15b cells revealed poor downregulation of both factors (Figure 2B, lower panels). Hence, in further experiments, only CFP15a cells were analyzed. The level of CFTR transcripts increased by $3.2 \pm 0.16$-fold in CFP15a cells in which UPF1 was downregulated relative to untreated cells (Figure 2C). Downregulation of UPF2, which was less efficient than that of $U P F 1$, also increased the transcript level, but to a lesser extent, by $1.7 \pm 0.20$-fold (Figure 2D). These results are consistent with the Western blot, which showed less efficient downregulation of UPF2 than of UPF1 (Figure 2B, upper panels). As a control, we treated the CFP22a cells with the UPF1 and UPF2 siRNA. As expected, no change in the CFTR transcript level was found (Figure 2, C and D). Together, the results suggest that NMD efficiency for transcripts carrying the W1282X mutation is different among different cell lines even derived from the same tissue.

Variable NMD efficiency of physiologic NMD substrates. We further investigated the possibility that the variability in NMD efficiency might be a general phenomenon and is not specific to CFTR transcripts carrying PTCs. For this we analyzed in the 3 epithelial cell lines (CFP15a, CFP15b, and CFP22a) the effect of CHX treatment on the level of physiologic NMD substrates. These included an alternatively spliced PTC-bearing transcript RPL3 (50); transcripts with introns in their $3^{\prime}$ UTR (the SC35 splicing isoforms $S C 351.6 \mathrm{~kb}$ and $S C 351.7 \mathrm{~kb})(51,52)$; a transcript with a uORF (ASNS) (33); and another bona fide NMD substrate with an unknown NMD-inducing feature, CARS (33). For all 5 transcripts analyzed, the results show an increase in the level following CHX treatment, which widely varied among the cells (Figure 3, A-E). In the CFP15b cells, the increase was significantly lower 
A

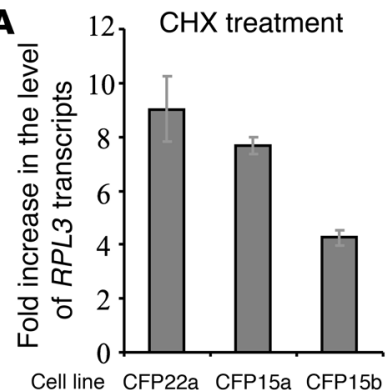

B

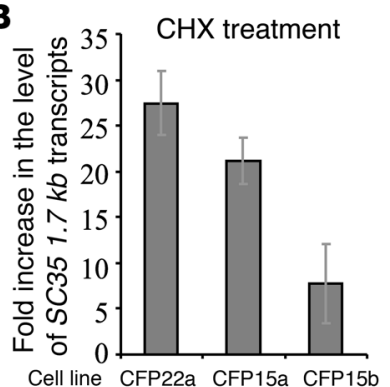

Cell line CFP22a CFP15a CFP15b

C

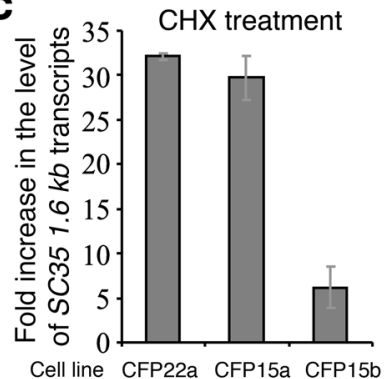

D

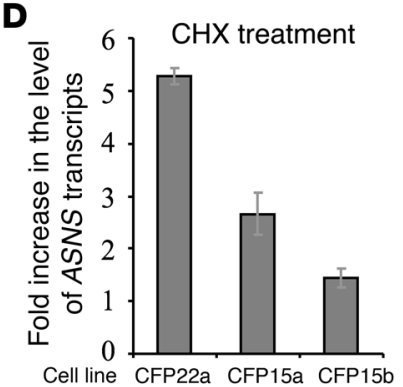

E

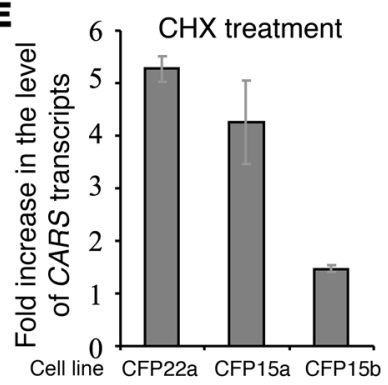

$\mathbf{F}$

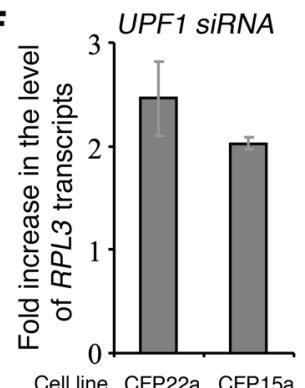

Cell line CFP22a CFP15a

G

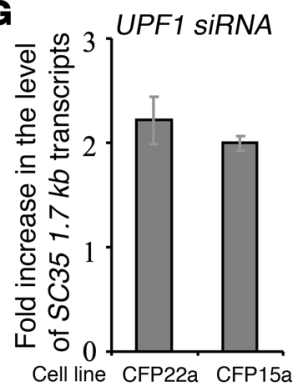

H

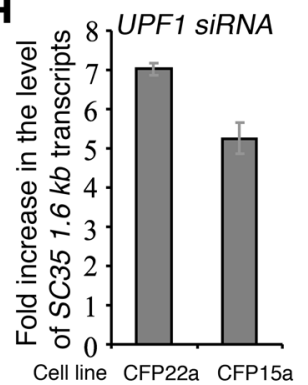

I

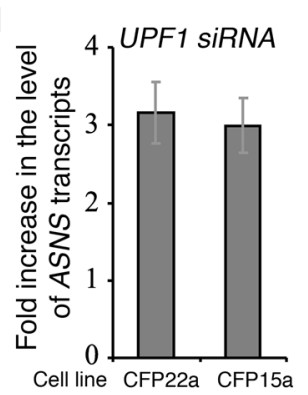

J

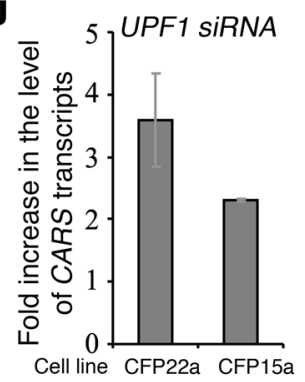

than that observed in the other cells. The level of these physiologic NMD substrates was further analyzed in CFP15a and CFP22a cells following UPF1 downregulation (Figure 3, F-J). As shown in Figure 3, the fold increase following CHX treatment was higher than that observed following UPF1 downregulation (Figure 3, compare A-E with F-J). We conjecture that this effect probably

\section{Figure 3}

Effect of NMD inhibition on the transcript level of physiologic NMD substrates in nasal epithelial cell lines. The level of each analyzed transcript was measured by real-time PCR and normalized to that of GAPDH and the ratio between these normalized levels following treatment was calculated and compared to the ratio in untreated cells. (A-E) Effect of indirect NMD inhibition by $\mathrm{CHX}$ on the level of RPL3 (A), SC35 $1.7 \mathrm{~kb}$ (B), SC35 $1.6 \mathrm{~kb}(\mathbf{C})$, ASNS (D), and CARS (E) transcripts. (F-J) Effect of direct NMD inhibition by UPF1 downregulation on the level of RPL3 (F), SC35 $1.7 \mathrm{~kb}(\mathbf{G})$, SC35 $1.6 \mathrm{~kb}(\mathbf{H})$, ASNS (I), and CARS $(\mathbf{J})$ transcripts. The increase in the level of CFTR transcripts is shown as mean \pm SEM.

resulted from the incomplete NMD inhibition following UPF1 downregulation. Variability in NMD efficiency following UPF1 downregulation was also found among other epithelial cell lines (unpublished observations). Together, the results following CHX treatment and UPF1 downregulation indicated a wide variability in NMD efficiency among different cell lines, even in cell lines derived from the same tissue.

We further analyzed the relative level of all 5 physiologic NMD substrates in RNA samples from nasal epithelial cells derived from 3 patients: 2,8 , and 10 . The results showed that the relative levels of all analyzed transcripts were lower in patient 10 than in patients 2 and 8 (Figure 4). This is consistent with the levels of the nonsense CFTR mRNA, which were lower in patient 10 than in patients 2 and 8 .

NMD governs the CFTR function following gentamicin treatment. Previous studies in Saccharomyces cerevisiae found that suppressor transfer RNA (which promotes readthrough) led to sufficient levels of functional proteins expressed from a nonsense allele only after inhibition of NMD (53-55). Here we aimed to investigate the potential of NMD inhibition to modulate the response to gentamicin in epithelial cells derived from CF patients. Since the CFTR gene encodes a cAMP-activated chloride channel, we analyzed the effect of NMD inhibition on the CFTR function by measuring cAMP-evoked chloride efflux. The results showed that the CFTR channels in CFP15a cells are inactive or absent (Figure 5A). Following treatment with $50-200 \mu \mathrm{g} / \mathrm{ml}$ gentamicin, concentrationdependent chloride efflux was detected (Figure 5A). This indicates that gentamicin can induce productive readthrough of the PTCs in these cells. Chloride channel activity was improved in gentamicin-treated cells in which UPF1 or UPF2 was downregulated compared with cells treated with gentamicin alone (Figure 5, C-E). The downregulation of UPF1 resulted in a higher activation of the CFTR channels relative to UPF2, consistent with the different efficiency of the downregulation of these factors (Figure 2B, upper panels). Interestingly, UPF1 downregulation in cells treated with $50 \mu \mathrm{g} / \mathrm{ml}$ gentamicin resulted in chloride efflux comparable to that achieved with $100 \mu \mathrm{g} / \mathrm{ml}$ gentamicin alone (Figure 5, C and D). Moreover, UPF1 downregulation in cells treated with $100 \mu \mathrm{g} / \mathrm{ml}$ resulted in an even higher activation compared with that achieved with 200 $\mu \mathrm{g} / \mathrm{ml}$ gentamicin alone (Figure 5, D and E). To a lesser extent, this effect was also observed following UPF2 downregulation. In order to evaluate the extent of functional improvement following NMD inhibition in CFP15a, we examined the CFTR function in human epithelial cells T84, which carry normal CFTR alleles (Figure 5B). The results show that the CFTR function is similar in T84 cells and CFP15a cells following UPF1 downregulation together with treatment with $200 \mu \mathrm{g} / \mathrm{ml}$ gentamicin (Figure 5, compare B and E). 

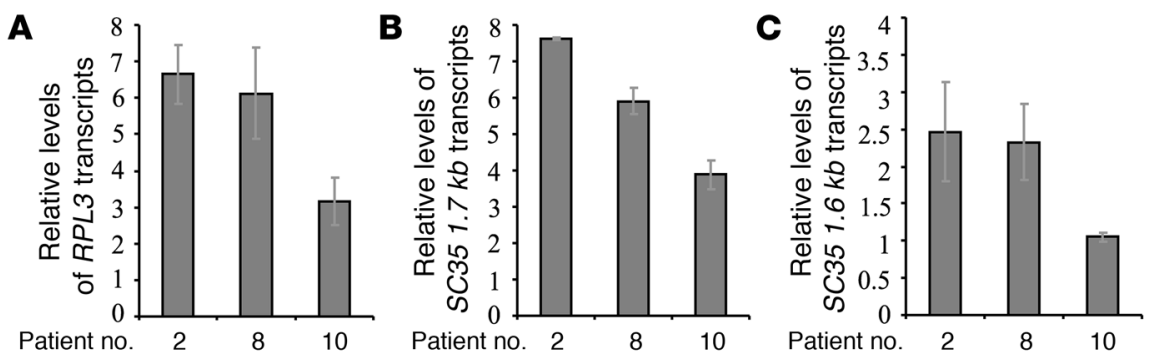

\section{Figure 4}

Relative levels of physiologic NMD substrates in RNA samples derived from CF patients. The level of each analyzed transcript - RPL3 (A), SC35 $1.7 \mathrm{~kb}$ (B), SC35 $1.6 \mathrm{~kb}$ (C), ASNS (D), and CARS (E) - was measured by real-time PCR and normalized to the level of $G A P D H$. The relative levels are shown as mean \pm SEM.
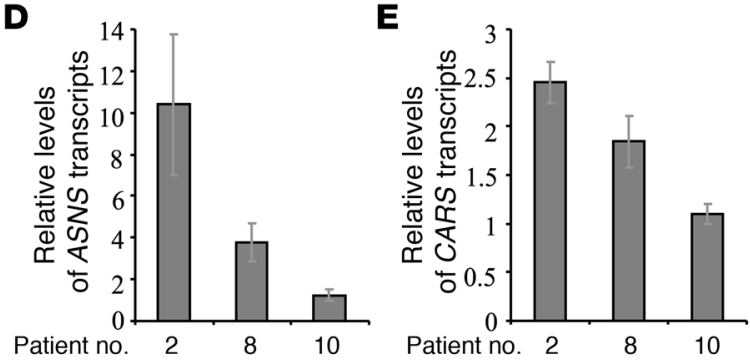

We extended the CFTR functional analysis to another epithelial cell line, IB3-1. Previous studies have shown that the level of the W1282X transcripts in these cells is markedly reduced in comparison to the level of transcripts derived from the $\Delta \mathrm{F} 508$ allele (5). As shown in Figure 5G, the CFTR channels in IB3-1 cells were inactive. No significant activation was observed following treatment of the cells with $50 \mu \mathrm{g} / \mathrm{ml}, 100 \mu \mathrm{g} / \mathrm{ml}$, or even $200 \mu \mathrm{g} / \mathrm{ml}$ gentamicin (Figure $5 \mathrm{G})$. Following downregulation of UPF1 together with treatment with $50 \mu \mathrm{g} / \mathrm{ml}$ gentamicin, no significant improvement in chloride efflux was achieved (Figure 5H). Only following downregulation of UPF1 together with treatment with $100 \mu \mathrm{g} / \mathrm{ml}$ or $200 \mu \mathrm{g} / \mathrm{ml}$ gentamicin was an impressive CFTR activation observed (Figure 5, I and J). It is important to note that this CFTR activation was found despite a nonefficient transfection ( 20\%) with UPF1 siRNA (data not shown). These results indicate that the CFTR activation in IB3-1 cells by gentamicin was highly dependent on NMD downregulation.

We also analyzed the CFTR function in the homozygous $\Delta F 508$ CFP22a cells. No CFTR chloride efflux was found in CFP22a cells following gentamicin treatment, as expected from cells carrying CFTR mutations with no potential for readthrough (Figure $5 \mathrm{~K}$ ). Furthermore, we found no improvement following downregulation of UPF1 or UPF2 (Figure $5 \mathrm{~K}$ ). This result is consistent with the inability of NMD inhibition to increase the $\triangle \mathrm{F} 508$ transcript level (Figure 2, C and D). These results support the general concept that NMD affects the level of nonsense transcripts and governs the response to gentamicin.

\section{Discussion}

Our study presents what we believe to be new insights into the molecular basis of the response to treatments that promote readthrough of PTCs by showing a potential role for the NMD mechanism in governing the response. Previous studies emphasized the contribution of cis-elements, such as the stop codon and the first nucleotide that follows, to the response to aminoglycoside treatment. Our results indicate that factors other than the PTC locus itself, such as the level of nonsense transcripts, might limit the readthrough efficiency. In cases of efficient NMD, the level of nonsense transcripts is markedly reduced and hence is insufficient to generate enough functional proteins even when gentamicin is provided. By contrast, in cases of less-efficient NMD, the transcript level is higher and readthrough might be effective. It was previously suggested that no clinical benefit would be derived from aminoglycoside treatment in CF patients with severely reduced levels of nonsense transcripts (56). Our results, although performed on a very small number of patients, support this hypothesis and show no correction of the CFTR function in patients with markedly lower levels of nonsense transcripts (Figure 1 and Table 1). The results further show that by increasing the level of CFTR nonsense transcripts, a significant improvement in the response to gentamicin can be achieved. This leads to increased activation of the CFTR channel compared with the activation with gentamicin alone (Figure 5). It follows that in the same cells, increase in the level of nonsense transcripts can allow the use of lower gentamicin concentrations to achieve chloride efflux activation similar to that achieved with gentamicin alone. Although the gentamicin concentrations used in this study were much higher than the clinical doses, the results provide a proof of concept that the level of PTC-bearing transcripts affects the response to readthrough treatment.

Here we show, for a variety of transcripts, that NMD efficiency might vary considerably among cells derived from the same cell type (epithelium) and even from the same tissue (nasal epithelium) (Figures 2 and 3 ). The results also clearly show that there are cell lines, such as CFP15b, in which NMD is relatively less efficient. It is important to note that in all cell lines, different transcripts are subject to different extents of NMD (Figure 3). Previously it was shown that in patients carrying PTCs in collagen X, the nonsense transcripts were subject to efficient NMD in cartilage cells, while in noncartilage cells, no NMD was observed (57). Recently, in 2 unrelated fetuses with Roberts syndrome, both homozygous for a PTC in the ESCO2 gene, different tissues showed different NMD efficiency (58). These studies suggest that the NMD efficiency of transcripts carrying PTCs might vary among different tissues. These differences probably result from the interaction between elements in transcripts that are subject to NMD and factors in the NMD machinery. Together, the results suggest that variability in NMD efficiency might be a more generalized phenomenon; however, further studies are needed.

Our results point to the possible role of NMD efficiency in regulation of normal cellular functions. Variable NMD might lead to 
A
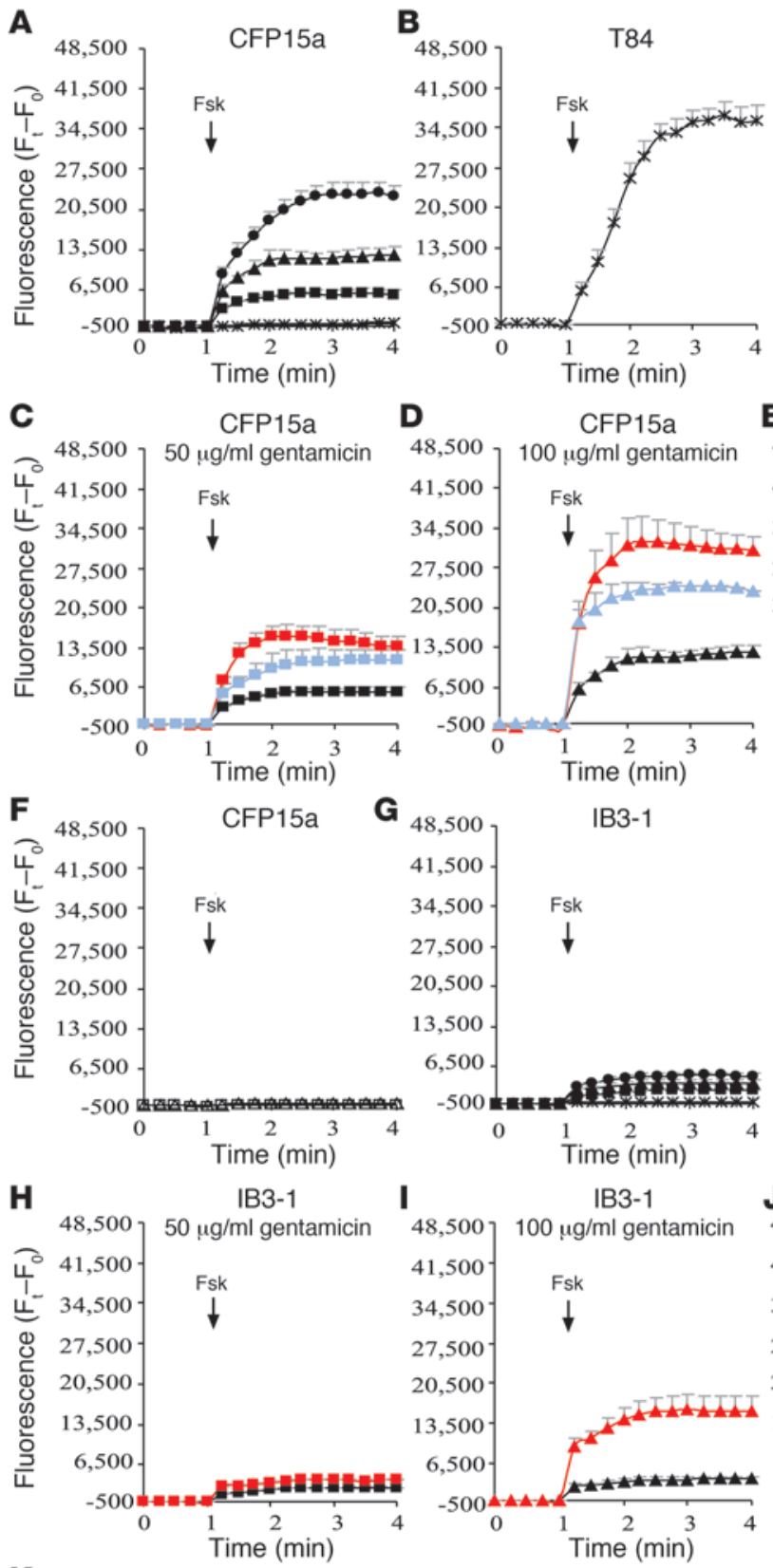

* Untreated cells

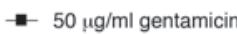

^ $100 \mu \mathrm{g} / \mathrm{ml}$ gentamicin

- $200 \mu \mathrm{g} / \mathrm{ml}$ gentamicin

Black Gentamicin alone

Red Gentamicin + UPF1 siRNA

Blue Gentamicin + UPF2 siRNA

- UPF1 SiRNA alone

$\triangle$ UPF2 siRnA alone
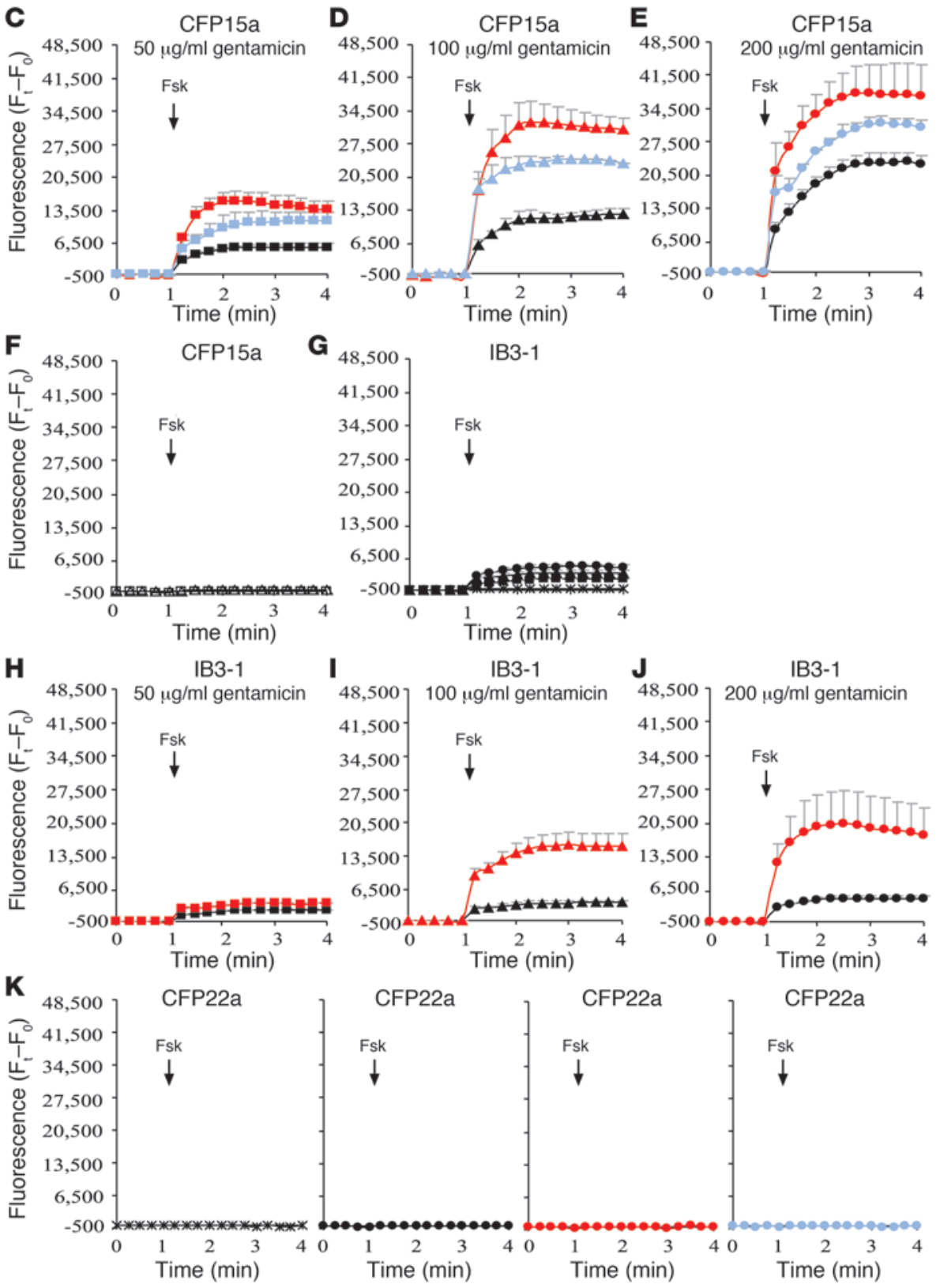

\section{Figure 5}

Effect of NMD downregulation on the CFTR chloride efflux following gentamicin treatment. (A) CFP15a cells treated with 50-200 $\mu \mathrm{g} / \mathrm{ml}$ gentamicin. (B) Chloride efflux in T84 cells. (C-E) CFP15a cells treated with siRNA directed against UPF1 and UPF2 following gentamicin treatment with $50 \mu \mathrm{g} / \mathrm{ml}$ (C), $100 \mu \mathrm{g} / \mathrm{ml}$ (D), and 200 $\mu \mathrm{g} / \mathrm{ml}(\mathbf{E})$. (F) CFP15a cells treated with siRNA directed against UPF1 or UPF2 alone. (G) IB3-1 cells treated with 50-200 $\mu \mathrm{g} / \mathrm{ml}$ gentamicin. $(\mathbf{H}-\mathbf{J})$ IB3-1 cells treated with siRNA directed against UPF1 following gentamicin treatment with $50 \mu \mathrm{g} /$ $\mathrm{ml}(\mathbf{H}), 100 \mu \mathrm{g} / \mathrm{ml}$ (I), and $200 \mu \mathrm{g} / \mathrm{ml}(\mathbf{J})$. (K) CFP22a cells treated with $200 \mu \mathrm{g} / \mathrm{ml}$ gentamicin alone and with siRNA directed against UPF1 and UPF2. Fsk, forskolin. The arrow indicates the time of forskolin administered. The increase in chloride efflux is shown as mean \pm SEM. $F_{t}$, fluorescence intensity at the reading time; $F_{0}$, fluorescence intensity at the beginning of the experiment.

or deleterious gain-of-function effect. For example, in $\beta$-thalassemia, the truncated proteins derived from transcripts carrying nonsense mutations cause toxic precipitation of insoluble globin chains, which might affect the phenotype of heterozygous patients (60).

It was previously suggested that readthrough-promoting reagents may not cause appreciable stabilization of nonsense transcripts due to low efficiency of readthrough or initiation of alternative surveillance mechanisms (61). In our study, we observed no increase in the level of CFTR nonsense transcripts following gentamicin treatment in patients and cell lines (Supplemental Figure 2). However, gentamicin concentration-dependent correction of the CFTR function was observed in the cells. It is possible that this resulted from low readthrough levels, which were sufficient for functional restoration (62). Similarly, another study in 2 cell lines, one compound heterozygous for nonsense alleles in the HEXB gene and the other monoallelic for a nonsense allele in TP53 gene, found no change in the level of nonsense transcripts following treatment with aminoglycosides (63). However, in IB3-1

differences in disease severity in the case of truncated proteins with partial function. This may explain the phenotypic variability among DMD and BMD patients carrying the same nonsense mutation in the dystrophin gene (59). Variable NMD efficiency might have an effect in cases where the truncated protein has a dominant-negative cells, transcript stabilization was observed following gentamicin treatment (5). Hence, further investigation of the potential for gentamicin to stabilize transcripts in vitro and in vivo is required.

The small number of primary cells scraped from the nasal epithelium of CF patients who participated in the clinical trial did not 
enable us to perform experiments aimed to directly investigate the role of NMD in response to gentamicin. However, the variability in the level of a variety of PTC-bearing transcripts suggests that the NMD mechanism might have contributed to the response of these patients to gentamicin treatment. It is worth noting that the role of posttranscriptional mechanisms in regulating the response to gentamicin cannot be excluded, except for alternative splicing of CFTR exon 20, which we found not be upregulated by the W1282X mutation (Supplemental Results and Supplemental Figure 4).

Finally, our results are important for the development of new therapeutic approaches aiming to promote readthrough of PTCs in many human genetic diseases. Such approaches should consider the possible effect of low levels of PTC-bearing transcripts on the response, although high transcript levels might not necessarily predict a response. It is interesting to note that we and others are currently investigating the effect of a newly developed small molecule, PTC124, on CF patients carrying the W1282X mutation (64). The role of NMD in regulating the response to this treatment is being studied in light of the results presented here.

\section{Methods}

Patients. Five of the patients were heterozygous for the W1282X and the $\Delta$ F508 mutations (patients 1-5); 3 were homozygous for the W1282X mutation (patients 6, 8, and 9); 1 was heterozygous for the W1282X and G542X mutations (patient 7); and 1 was heterozygous for W1282X and $3849+10 \mathrm{~kb} \mathrm{C} \rightarrow \mathrm{T}$ mutations (patient 10), which can lead to inclusion of an 84-bp cryptic exon harboring a PTC. The clinical data of the patients and the protocol of the double blind placebo-controlled crossover trial were previously described (29).

Basal potential difference and chloride transport measured by NPD. NPD was measured in the nasal epithelium of patients before treatment and after placebo and gentamicin treatment, as described in Wilschanski et al. (29). In brief, measurements were performed between a fluid-filled recording bridge on the nasal mucosa and a reference bridge. In this way, consistent baseline measurements of potential difference were obtained. Change in the voltage response, which indicates nasal chloride permeability, was measured following generation of a large chloride chemical gradient across the apical membrane.

Cell cultures. Nasal epithelial cell lines were established from nasal polyps of CF patients using E6/E7 genes of the human papilloma virus 18 and hTERT for CFP22a, E6/E7 genes alone for CFP15a, and LT-hTERT for CFP15b $(65,66)$. These cells were grown in bronchial epithelial cell basal medium (Cambrex). T84 cells, which were derived from lung metastases from colorectal carcinoma, were grown in DMEM-F12 supplemented with $10 \%$ FCS. IB3-1 cells, which were derived from a primary culture of bronchial epithelia isolated from a CF patient (67), were grown in DMEM supplemented with $10 \%$ FCS.

RNA analysis. Scraped nasal epithelial cells were obtained from the patients before treatment and after treatment with placebo or gentamicin. Total RNA was extracted using the RNeasy Extraction kit (QIAGEN) or TriReagent-LS (Molecular Research Center). Reactions without RNA and RT were used as controls. The level of the nonsense CFTR mRNA in samples of W1282X/AF508 patients was analyzed by RT-PCR with primers in exons 10 and 11 , flanking the $\Delta \mathrm{F} 508$ mutation. The $\Delta \mathrm{F} 508$ mutation is a 3-bp deletion which is not expected to be affected by NMD, hence the level of $\Delta \mathrm{F} 508$ transcripts is similar to that of wild type (38). Thus, the level of mRNA transcribed from W1282X alleles could be distinguished and compared to those transcribed from the $\triangle \mathrm{F} 508$ allele. Sequences for the primer pair used were as follows: forward GATTATGGGAGAACTGGAGC, reverse TTCTTGCTCGTTGACCTCCA. The forward primer was fluorescently labeled with 6-FAM. The PCR products of the $\triangle \mathrm{F} 508$ transcripts and the W1282X transcripts were 254 and $257 \mathrm{bp}$, respectively. The conditions were determined by serial tertiary dilutions and by the number of cycles. Each PCR product $(1 \mu \mathrm{l})$ was mixed with $0.3 \mu \mathrm{l}$ of a 500-TAMRA-labeled commercial size standard (Applied Biosystems) and run on an ABI PRISM 377 system (Applied Biosystems). The analysis was performed using GeneScan software (version 3.1; Applied Biosystems). The level of the W1282X transcripts was determined as the peak area of the signal of the W1282X PCR product divided by the peak area of the signal of the $\triangle F 508$ PCR product.

For the RNA samples of the other 5 patients and of CFP15a, CFP15b, and CFP22a cell lines, we performed real-time PCR in the LightCycler (software version 3.5) using a FastStart DNA Master SYBR Green I kit (Roche Diagnostics). The CFTR mRNA levels were normalized to those of mRNA transcribed from a control gene, RPS9. For each pair of primers, a standard curve was derived, and annealing temperature was optimized in order to exclude PCR artifacts. Moreover, we included in the analysis only experiments in which the standard curves were as expected, to ensure accurate quantification. The level of the normalized CFTR nonsense mRNA in each patient was compared with the normalized level obtained for a control individual with normal CFTR alleles. For CFP15a, CFP15b, and CFP22a cells, the level of the normalized CFTR transcripts was compared to the normalized level of untreated cells. Sequences of the primer pairs used were as follows: CFTR mRNA: forward, GAGGGTAAAATTAAGCACAGT, reverse, TGCTCGTTGACCTCCA; RPS9: forward AGACCCTTCGAGAAATCTCGTCTCG, reverse TGGGTCCTTCTCATCAAGCGTCAGC.

For the RNA samples of the patients carrying 2 nonsense mutations, we also normalized the CFTR level to the level of KRT18, a gene expressed specifically at ciliated and secretory epithelial cells. Quantification analyses were performed using 2 methods: real-time PCR and GeneScan. Real-time PCR reactions were performed as with the RPS9 (see above). For the GeneScan analysis, the CFTR and KRT18 transcripts were amplified in a multiplex PCR. Two different fluorescently labeled forward primers were used: 6-FAM for CFTR forward primer and HEX for KRT18 forward primer. CFTR primer pair used here was the same as that used for the reactions with samples from the $W 1282 X / \Delta F 508$ patients. Sequences of the KRT18 primer pair were: forward AGTCTGTGGAGAACGACATCC, reverse TGGTGCTCTCCTCAATCTGC. The PCR products of the CFTR transcripts were $257 \mathrm{bp}$, and those of KRT18 were $311 \mathrm{bp}$. Similar transcript levels were obtained using either of these quantification methods.

Analysis of the level of CFTR pre-mRNA was performed using the realtime PCR system, using primers in intron 5 of the gene. Two-tailed Student's $t$ test was used to analyze the differences in CFTR pre-mRNA levels between patients 5 and 10. $P$ values less than 0.05 were considered to be significant. The sequences of the primer pair were: CFTR pre-mRNA: forward CTCAGCTCTAGCTTCCC, reverse GCTCAGGTATCATATCTGGC. All RT-PCR experiments were repeated at least 3 times, for both patient and cell line samples.

For quantification of physiologic NMD substrates, RPL3, SC35 $1.6 \mathrm{~kb}$, $S C 351.7 \mathrm{~kb}$, ASNS, and CARS, we also performed real-time PCR. The levels of each transcript were normalized to the levels of GAPDH transcripts. In the samples from cell lines, the normalized level of the transcripts following NMD inhibition was compared with the normalized level of untreated cells. Sequences of the primer pairs used were as follows: RPL3: forward GGCATTGTGGGCTACGTG, reverse CTTCAGGAGCAGAGCAGA; $S C 351.6 \mathrm{~kb}$ : forward CGGTGTCCTCTTAAGAAAATGATGTA, reverse CTGCTACACAACTGCGCCTTTT; SC35 $1.7 \mathrm{~kb}$ : forward GGCGTGTATTGGAGCAGATGTA, reverse: the same as for SC35 $1.6 \mathrm{~kb}$; ASNS: forward TCAGGTTGATGATGCAATG, reverse CAGCTGACTTGTAGTGGGTC; CARS: forward AAATTAAATGAGACCACGGA, reverse TGACATCACAGCCAAGTGTA; GAPDH: forward TGAGCTTGACAAAGTGGTCG, reverse GGCTCTCCAGAACATCATCC. In order to analyze the splicing pattern 
of the W1282X region, we performed RT-PCR reactions with primers in exons 18 and 21: forward GTAAACTCCAGCATAGATGTGG, reverse CCACTGTTCATAGGGATCCAA. The expected PCR product from normal splicing in this region was $492 \mathrm{bp}$.

Gentamicin treatment and NMD inbibition. Cells were treated with 50-200 $\mu \mathrm{g} / \mathrm{ml}$ gentamicin sulphate (Biological Industries) for 18-24 hours. We indirectly inhibited NMD by treating the cells with $200 \mu \mathrm{g} / \mathrm{ml} \mathrm{CHX}$ (Sigma-Aldrich) for 5 hours. siRNA oligonucleotides for silencing UPF1 and UPF2 expression (Dharmacon) were those described by Mendell et al. (49). siRNA oligonucleotide for luciferase (Dharmacon) was described by Gehring et al. (68). Nonspecific control oligonucleotide (Dharmacon) contained 52\% GC content, similar to the GC content in both specific oligonucleotides. $10^{5}$ cells were plated for RNA analysis into 6-well plates and for protein analysis into $100-\mathrm{mm}$ plates, 24 hours before transfection. $10^{4}$ cells were plated for functional analysis into 96-well plates, 72 hours before transfection. Two hundred nanomolar of each RNAi oligonucleotide was transfected using Oligofectamine (Invitrogen). Cells were further grown for 24-48 hours. Gentamicin was added for 18-24 hours before the cells were harvested for RNA or protein analysis or for measurements of CFTR function. Experiments were repeated at least 3 times.

Western blot analysis. Total proteins $(20-40 \mu \mathrm{g})$ were extracted from the different cell lines and were subjected to SDS-PAGE as previously described (68). These proteins were probed with antibodies against $U P F 1, U P F 2$, and $\beta$-catenin.

CFTR functional analysis. CFTR function was measured by chloride $\left(\mathrm{Cl}^{-}\right)$ efflux, using $\mathrm{N}$-(6-methoxyquinolyl) acetoethyl ester (MQAE), a $\mathrm{Cl}^{-}$-sensitive fluorescence indicator (Invitrogen). Cells were loaded overnight with MQAE. The rate of $\mathrm{Cl}^{-}$efflux was measured in response to exchange of extracellular $\mathrm{Cl}^{-}$with nitrate $\left(\mathrm{NO}_{3}^{-}\right)$, an anion that passes through the CFTR but unlike $\mathrm{Cl}^{-}$does not quench the indicator's fluorescence. Activation of the CFTR chloride channel was stimulated by the CAMP agonist forskolin. The fluorescence measurements were performed using the FLUOstar Galaxy fluorescence reader (BMG LABTECH). For evaluation of CFTR activation, the difference between fluorescence intensity at the reading time $\left(\mathrm{F}_{\mathrm{t}}\right)$ and that at the beginning of the experiment $\left(\mathrm{F}_{0}\right)$ was calculated.

\section{Acknowledgments}

We thank all CF centers in Israel, especially J. Rivlin, Y. Yahav, H. Blau, L. Bentur, and M. Aviram. We also thank J.T. Mendell and J. Lykke-Andersen for UPF1 and UPF2 antibodies; B. Shanitzki for assistance with the RNA quantification analyses; and M. Viegas for primers for ASNS and CARS transcripts. The work was partially supported by a grant from Yissum to Batsheva Kerem and a Deutsche Forschungsgemeinschaft grant to Andreas E. Kulozik.

Received for publication March 14, 2006, and accepted in revised form December 12, 2006.

Address correspondence to: Batsheva Kerem, Department of Genetics, The Life Sciences Institute, The Hebrew University, Jerusalem, Israel 91904. Phone: 972-2-6585689; Fax: 972-2-6584810; E-mail: kerem@cc.huji.ac.il.

Stephanie Boelz and Malka Nissim-Rafinia contributed equally to this work.
1. Frischmeyer, P.A., and Dietz, H.C. 1999. Nonsensemediated mRNA decay in health and disease. Hum. Mol. Genet. 8:1893-1900.

2. Burke, J.F., and Mogg, A.E. 1985. Suppression of a nonsense mutation in mammalian cells in vivo by the aminoglycoside antibiotics G-418 and paromomycin. Nucleic Acids Res. 13:6265-6272.

3. Martin, R., Mogg, A.E., Heywood, L.A., Nitschke, L., and Burke, J.F. 1989. Aminoglycoside suppression at UAG, UAA and UGA codons in Escherichia coli and human tissue culture cells. Mol. Gen. Genet. 217:411-418.

4. Howard, M., Frizzell, R.A., and Bedwell, D.M. 1996. Aminoglycoside antibiotics restore CFTR function by overcoming premature stop mutations. Nat. Med. 2:467-469.

5. Bedwell, D.M., et al. 1997. Suppression of a CFTR premature stop mutation in a bronchial epithelial cell line. Nat. Med. 3:1280-1284.

6. Barton-Davis, E.R., Cordier, L., Shoturma, D.I., Leland, S.E., and Sweeney, H.L. 1999. Aminoglycoside antibiotics restore dystrophin function to skeletal muscles of mdx mice. J. Clin. Invest. 104:375-381.

7. Sleat, D.E., Sohar, I., Gin, R.M., and Lobel, P. 2001. Aminoglycoside-mediated suppression of nonsense mutations in late infantile neuronal ceroid lipofuscinosis. Eur. J. Paediatr. Neurol. 5(Suppl. A):57-62.

8. Keeling, K.M., et al. 2001. Gentamicin-mediated suppression of Hurler syndrome stop mutations restores a low level of alpha-L-iduronidase activity and reduces lysosomal glycosaminoglycan accumulation. Hum. Mol. Genet. 10:291-299.

9. Zsembery, A., et al. 2002. Correction of CFTR malfunction and stimulation of Ca-activated $\mathrm{Cl}$ channels restore $\mathrm{HCO} 3$ - secretion in cystic fibrosis bile ductular cells. Hepatology. 35:95-104.

10. Schulz, A., et al. 2002. Aminoglycoside pretreatment partially restores the function of truncated $\mathrm{V}(2)$ vasopressin receptors found in patients with nephrogenic diabetes insipidus. J. Clin. Endocrinol. Metab. 87:5247-5257.
11. Helip-Wooley, A., Park, M.A., Lemons, R.M., and Thoene, J.G. 2002. Expression of CTNS alleles: subcellular localization and aminoglycoside correction in vitro. Mol. Genet. Metab. 75:128-133.

12. Du, M., et al. 2002. Aminoglycoside suppression of a premature stop mutation in a Cftr-/- mouse carrying a human CFTR-G542X transgene. J. Mol. Med. 80:595-604.

13. Arakawa, M., et al. 2003. Negamycin restores dystrophin expression in skeletal and cardiac muscles of mdx mice. J. Biochem. (Tokyo). 134:751-758.

14. Sangkuhl, K., et al. 2004. Aminoglycoside-mediated rescue of a disease-causing nonsense mutation in the $\mathrm{V} 2$ vasopressin receptor gene in vitro and in vivo. Hum. Mol. Genet. 13:893-903.

15. Howard, M.T., et al. 2004. Readthrough of dystrophin stop codon mutations induced by aminoglycosides. Ann. Neurol. 55:422-426.

16. Bidou, L., et al. 2004. Premature stop codons involved in muscular dystrophies show a broad spectrum of readthrough efficiencies in response to gentamicin treatment. Gene Ther. 11:619-627.

17. Hein, L.K., et al. 2004. alpha-L-iduronidase premature stop codons and potential read-through in mucopolysaccharidosis type I patients. J. Mol. Biol. 338:453-462.

18. Lai, C.H., et al. 2004. Correction of ATM gene function by aminoglycoside-induced read-through of premature termination codons. Proc. Natl. Acad. Sci.U.S. A. 101:15676-15681.

19. Wolstencroft, E.C., Mattis, V., Bajer, A.A., Young, P.J., and Lorson, C.L. 2005. A non-sequence-specific requirement for SMN protein activity: the role of aminoglycosides in inducing elevated SMN protein levels. Hum. Mol. Genet. 14:1199-1210.

20. Wagner, K.R., et al. 2001. Gentamicin treatment of Duchenne and Becker muscular dystrophy due to nonsense mutations. Ann. Neurol. 49:706-711.

21. Politano, L., et al. 2003. Gentamicin administration in Duchenne patients with premature stop codon. Preliminary results. Acta Myol. 22:15-21.
22. Wilschanski, M., et al. 2000. A pilot study of the effect of gentamicin on nasal potential difference measurements in cystic fibrosis patients carrying stop mutations. Am. J. Respir. Crit. Care Med. 161:860-865.

23. Clancy, J.P., et al. 2001. Evidence that systemic gentamicin suppresses premature stop mutations in patients with cystic fibrosis. Am. J. Respir. Crit. Care Med. 163:1683-1692.

24. Grayson, C., et al. 2002. In vitro analysis of aminoglycoside therapy for the Arg120stop nonsense mutation in RP2 patients. J. Med. Genet. 39:62-67.

25. Dunant, P., Walter, M.C., Karpati, G., and Lochmuller, H. 2003. Gentamicin fails to increase dystrophin expression in dystrophin-deficient muscle. Muscle Nerve. 27:624-627.

26. Howard, M.T., et al. 2000. Sequence specificity of aminoglycoside-induced stop codon readthrough: potential implications for treatment of Duchenne muscular dystrophy. Ann. Neurol. 48:164-169.

27. Manuvakhova, M., Keeling, K., and Bedwell, D.M. 2000. Aminoglycoside antibiotics mediate contextdependent suppression of termination codons in a mammalian translation system. RNA. 6:1044-1055.

28. Keeling, K.M., and Bedwell, D.M. 2002. Clinically relevant aminoglycosides can suppress diseaseassociated premature stop mutations in the IDUA and P53 cDNAs in a mammalian translation system. J. Mol. Med. 80:367-376.

29. Wilschanski, M., et al. 2003. Gentamicin-induced correction of CFTR function in patients with cystic fibrosis and CFTR stop mutations. N. Engl. J. Med. 349:1433-1441.

30. Shoshani, T., et al. 1992. Association of a nonsense mutation (W1282X), the most common mutation in the Ashkenazi Jewish cystic fibrosis patients in Israel, with presentation of severe disease. Am. J. Hum. Genet. 50:222-228.

31. Maquat, L.E. 2004. Nonsense-mediated mRNA decay: splicing, translation and mRNP dynamics. Nat. Rev. Mol. Cell Biol. 5:89-99. 
32. Holbrook, J.A., Neu-Yilik, G., Hentze, M.W., and Kulozik, A.E. 2004. Nonsense-mediated decay approaches the clinic. Nat. Genet. 36:801-808.

33. Mendell, J.T., Sharifi, N.A., Meyers, J.L., MartinezMurillo, F., and Dietz, H.C. 2004. Nonsense surveillance regulates expression of diverse classes of mammalian transcripts and mutes genomic noise. Nat. Genet. 36:1073-1078.

34. Lewis, B.P., Green, R.E., and Brenner, S.E. 2003. Evidence for the widespread coupling of alternative splicing and nonsense-mediated mRNA decay in humans. Proc. Natl. Acad. Sci. U. S. A. 100:189-192.

35. He, F., et al. 2003. Genome-wide analysis of mRNAs regulated by the nonsense-mediated and $5^{\prime}$ to $3^{\prime}$ mRNA decay pathways in yeast. Mol. Cell. 12:1439-1452

36. Mitrovich, Q.M., and Anderson, P. 2000. Unproductively spliced ribosomal protein mRNAs are natural targets of mRNA surveillance in C. elegans. Genes Dev. 14:2173-2184.

37. Morrison, M., Harris, K.S., and Roth, M.B. 1997. smg mutants affect the expression of alternatively spliced SR protein mRNAs in Caenorhabditis elegans. Proc. Natl. Acad. Sci. U. S. A. 94:9782-9785

38. Trapnell, B.C., et al. 1991. Expression of the cystic fibrosis transmembrane conductance regulator gene in the respiratory tract of normal individuals and individuals with cystic fibrosis. Proc. Natl. Acad. Sci. U. S. A. 88:6565-6569.

39. Dupuit, F., et al. 1995. CFTR and differentiation markers expression in non-CF and $\Delta \mathrm{F} 508$ homozygous CF nasal epithelium. J. Clin. Invest. 96:1601-1611.

40. Wilschanski, M., et al. 2001. Nasal potential difference measurements in patients with atypical cystic fibrosis. Eur. Respir. J. 17:1208-1215.

41. Rubenstein, R.C., and Zeitlin, P.L. 1998. A pilot clinical trial of oral sodium 4-phenylbutyrate (Buphenyl) in deltaF508-homozygous cystic fibrosis patients: partial restoration of nasal epithelial CFTR function. Am. J. Respir. Crit. Care Med. 157:484-490.

42. Zeitlin, P.L., et al. 2002. Evidence of CFTR function in cystic fibrosis after systemic administration of 4-phenylbutyrate. Mol. Ther. 6:119-126.

43. McCarty, N.A., et al. 2002. A phase I randomized, multicenter trial of CPX in adult subjects with mild cystic fibrosis. Pediatr. Pulmonol. 33:90-98.
44. Zeitlin, P.L., Boyle, M.P., Guggino, W.B., and Molina, L. 2004. A phase I trial of intranasal Moli1901 for cystic fibrosis. Chest. 125:143-149.

45. Hamosh, A., Rosenstein, B.J., and Cutting, G.R. 1992 CFTR nonsense mutations G542X and W1282X associated with severe reduction of CFTR mRNA in nasal epithelial cells. Hum. Mol. Genet. 1:542-544.

46. Nagy, E., and Maquat, L.E. 1998. A rule for termination-codon position within intron-containing genes: when nonsense affects RNA abundance. Trends Biochem. Sci. 23:198-199.

47. Highsmith, W.E., et al. 1994. A novel mutation in the cystic fibrosis gene in patients with pulmonary disease but normal sweat chloride concentrations. N. Engl. J. Med. 331:974-980.

48. Carter, M.S., et al. 1995. A regulatory mechanism that detects premature nonsense codons in T-cell receptor transcripts in vivo is reversed by protein synthesis inhibitors in vitro. J. Biol. Chem. 270:28995-29003.

49. Mendell, J.T., ap Rhys, C.M., and Dietz, H.C. 2002. Separable roles for rent $1 / \mathrm{hUpf} 1$ in altered splicing and decay of nonsense transcripts. Science. 298:419-422.

50. Cuccurese, M., Russo, G., Russo, A., and Pietropaolo, C. 2005. Alternative splicing and nonsense-mediated mRNA decay regulate mammalian ribosomal gene expression. Nucleic Acids Res. 33:5965-5977.

51. Sureau, A., Gattoni, R., Dooghe, Y., Stevenin, J., and Soret, J. 2001. SC35 autoregulates its expression by promoting splicing events that destabilize its mRNAs. EMBO J. 20:1785-1796.

52. Gehring, N.H., et al. 2005. Exon-junction complex components specify distinct routes of nonsensemediated mRNA decay with differential cofactor requirements. Mol. Cell. 20:65-75.

53. Culbertson, M.R., Underbrink, K.M., and Fink, G.R. 1980. Frameshift suppression in Saccharomyces cerevisiae. II. Genetic properties of group II suppressors. Genetics. 95:833-853.

54. Leeds, P., Peltz, S.W., Jacobson, A., and Culbertson, M.R. 1991. The product of the yeast UPF1 gene is required for rapid turnover of mRNAs containing a premature translational termination codon. Genes Dev. 5:2303-2314.

55. Leeds, P., Wood, J.M., Lee, B.S., and Culbertson, M.R. 1992. Gene products that promote mRNA turnover in Saccharomyces cerevisiae. Mol. Cell. Biol. 12:2165-2177.

56. Dietz, H.C., and Hamosh, A. 1996. Nonstop treatment of cystic fibrosis. Nat. Med. 2:608-609.

57. Bateman, J.F., Freddi, S., Nattrass, G., and Savarirayan, R. 2003. Tissue-specific RNA surveillance? Nonsense-mediated mRNA decay causes collagen X haploinsufficiency in Schmid metaphyseal chondrodysplasia cartilage. Hum. Mol. Genet. 12:217-225.

58. Resta, N., et al. 2006. A homozygous frameshift mutation in the ESCO2 gene: evidence of intertissue and interindividual variation in Nmd efficiency. J. Cell. Physiol. 209:67-73.

59. Kerr, T.P., Sewry, C.A., Robb, S.A., and Roberts, R.G. 2001. Long mutant dystrophins and variable phenotypes: evasion of nonsense-mediated decay? Hum. Genet. 109:402-407.

60. Thein, S.L., et al. 1990. Molecular basis for dominantly inherited inclusion body beta-thalassemia. Proc. Natl. Acad. Sci. U. S. A. 87:3924-3928.

61. Frischmeyer, P.A., et al. 2002. An mRNA surveillance mechanism that eliminates transcripts lacking termination codons. Science. 295:2258-2261.

62. Farmen, S.L., et al. 2005. Gene transfer of CFTR to airway epithelia: low levels of expression are sufficient to correct $\mathrm{Cl}^{-}$transport and overexpression can generate basolateral CFTR. Am. J. Physiol. Lung Cell. Mol. Physiol. 289:L1123-L1130.

63. Noensie, E.N., and Dietz, H.C. 2001. A strategy for disease gene identification through nonsensemediated mRNA decay inhibition. Nat. Biotechnol. 19:434-439.

64. Ainsworth, C. 2005. Nonsense mutations: running the red light. Nature. 438:726-728.

65. Lundberg, A.S., et al. 2002. Immortalization and transformation of primary human airway epithelial cells by gene transfer. Oncogene. 21:4577-4586.

66. Yankaskas, J.R., et al. 1993. Papilloma virus immortalized tracheal epithelial cells retain a well-differentiated phenotype. Am. J. Physiol. 264:C1219-C1230.

67. Zeitlin, P.L., et al. 1991. A cystic fibrosis bronchial epithelial cell line: immortalization by adeno-12-SV40 infection. Am. J. Respir. Cell Mol. Biol. 4:313-319.

68. Gehring, N.H., Neu-Yilik, G., Schell, T., Hentze, M.W., and Kulozik, A.E. 2003. Y14 and hUpf3b form an NMD-activating complex. Mol. Cell. 11:939-949. 